\title{
Mir- 183-96-182 cluster may not work together in bovine ovarian granulosa cell apoptosis
}

Keywords: mir-183-96-182 cluster, cattle, granulosa cell, follicle, apoptosis

\section{Introduction}

Follicle development in cattle undergoes two important precesses, one is ovulation of the preovulatory dominant follicle, and the other is degeneration of anovulatory subordinate follicles Fortune et al. ${ }^{1}$ The degeneration of anovulatory subordinate follicles also called follicular atresia, in which apoptosis of granulosa cell is the main mechanism. ${ }^{1,2}$ miRNAs which are -22nt in length non-coding endogenous RNA molecules regulate gene expression in a sequence-dependent manner at posttranscriptional level Ambros. ${ }^{3}$ How these small molecules posttranscriptionally regulate apoptosis process and finally the follicles goes atresia is poorly understood. In our previous paper, miR-183 miRNA cluster which includes miR-183, miR-96 and miR-182 showed higher expression levels in dominant bovine follicles than in subordinates Ma et al. ${ }^{4}$ In this study, the miR-183-96-182 cluster will be transfected into bGCs separately to summary the functions of this cluster in apoptosis.

\section{Animal and GC collection}

The ovaries were collected in local abattoir. The classify of the ovaries is based on the rules described by Ireland et al..$^{5}$ Follicles $\leq 11$ and $\geq 12 \mathrm{~mm}$ in diameter were classified as subordinate and dominant, respectively Spicer et al. ${ }^{6}$ The GCs were collected in the follicles before ovulation. The oocytes were removed using a mouth-operated micropipette under a microscope, and the GCs were resuspended in DMEM medium with $10 \%(\mathrm{~V} / \mathrm{V})$ Fetal Bovine Serum.

\section{Apoptosis analysis of GCs}

The transfecion process is followed the Lipofectamine 2000 protocol. The $100 \mu \mathrm{M}$ miR-183, miR-96 and miR-182 mimics or control was tranfected into 12 -well culture plate. After $48 \mathrm{~h}$, the cells were enzymatically digested and washed with PBS. Then, the cells were measured through AnnexinV-FITC/PI double staining using the Annexin V-FITC Apoptosis Detection Kit (BD Biosciences Pharmingen) in accordance with the manufacturer's instructions. Results were analyzed using cell flow cytometry.

\section{Statistical analysis}

All experiments for miR-183-96-182 cluster or the control were conducted in triplicates. All data are presented as means \pm SE, and statistical evaluation of the data was conducted with SPSS 14.0. Statistical analysis was performed with the independent-sample Student's t-test to compare the two groups. Differences at $\mathrm{P}<0.05$ were considered statistically significant. To test the potential role of miR-183 members in GC apoptosis of the bovine follicles, the isolated bGCs were incubated in vitro and transiently transfected with miRNA mimics or NC. The apoptotic rate was estimated by Annexin V-FITC/PI double staining. Results indicated that miR-183-96-182 did not have the same trend in regulation of GC apoptosis, miR-183

\author{
Volume 6 Issue 2 - 2017 \\ Teng-He Ma, Gui-Da Ma, Xiao-Yi Gao, Zhi- \\ Chao Zhao, Yan-Wei Liu, Bin Wang, Yu-Xiang \\ Shi \\ Hebei University of Engineering, Handan city, China
}

Correspondence: Teng-He Ma, Hebei University of Engineering, Handan city, China, Email matenghe@।26.com

Received: April 06, 2017 | Published: December 0I, 2017

had no function to induce the apoptosis of bGCs $(\mathrm{P}>0.05)$, miR-182 and miR-96 have the opposite result, miR-183 could increase the apoptosis rates of bGCs $(\mathrm{P}<0.01)$, while miR-96 could decrease the apoptosis rates of bGCs $(\mathrm{P}<0.05)$.

miR-183-96-182 cluster had the function in regulation of cell apoptosis Tang et al. $^{7}$ and proliferation Zhang et al. ${ }^{8}$ With the $\mathrm{GC}$ aspirated from small growing follicles $(3-5 \mathrm{~mm}$ in diameter), Gebremedhn showed overexpression of miR-183-96-182 cluster promotes GC proliferation, they did not check the apoptosis rate of GC Gebremedhn et al. ${ }^{9}$ In our study, we did not find this cluster work together in apoptosis regulation, the reason needs further study. In conclusion, miR-183-96-182 cluster has diversiform regulation function in $\mathrm{GC}$ apoptosis, which may help to provide new clues in the research of follicular atresia.

\section{Conclusion}

In this study, cell flow cytometry was emplyed to test the function of miR-183-96-182 cluster in bovine ovarian granulosa cell apoptosis. miR-183-96-182 did not have the same trend in regulation of GC apoptosis, miR-183 had no function to induce the apoptosis of bGCs, miR-182 and miR-96 have the opposite result, miR-183 could increase the apoptosis rates of bGCs, while miR-96 could decrease the apoptosis rates of bGCs.

\section{Acknowledgements}

This study was supported by Natural Science Foundation of Hebei province (Grant No. C2016402061), Youth Foundation of Hebei Education Department (Grant No.QN2015012) and Doctoral Scientific Research Foundation, Hebei University of Engineering (Grant No.20120139).

\section{Conflict of interest}

Author declares that there is no conflict of interest.

\section{References}

1. Fortune JE, Sirois J, Turzillo AM, et al. Follicle selection in domestic ruminants. J Reprod Fertil. 1991;Suppl 43:187-198. 
2. Hernández-Coronado CG, Guzmán A, Espinosa-Cervantes R, et al. Sphingosine-1-phosphate and ceramide are associated with health and atresia of bovine ovarian antral follicles. Animal. 2015;9(2):308-312.

3. Ambros V. microRNAs: tiny regulators with great potential. Cell. 2001;107(7):823-826

4. Ma TH, Cheng L, Wang B, et al. Expression of the mir-183 cluster in the follicles and corpus luteum of cattle. Indian Journal of Animal Sciences. 2016;86(10):1153-1154.

5. Ireland JJ, Murphee RL, Coulson PB. Accuracy of predicting stages of bovine estrous cycle by gross appearance of the corpus luteum. J Dairy Sci. 1980;63(1):155-160.
6. Spicer LJ, Schreiber NB, Lagaly DV, et al. Effect of resistin on granulosa and theca cell function in cattle. Anim Reprod Sci. 2011;124(1-2):19-27.

7. Tang X, Zheng D, Hu P, et al. Glycogen synthase kinase 3 beta inhibits microRNA-183-96-182 cluster via the beta-Catenin/TCF/LEF-1 pathway in gastric cancer cells. Nucleic Acids Res. 2014;42(5):2988-2998.

8. Zhang Q, Ren W, Huang B, et al. MicroRNA-183/182/96 cooperatively regulates the proliferation of colon cancer cells. Mol Med Rep. 2015;12(1):668-674.

9. Gebremedhn S, Salilew-Wondim D, Hoelker M, et al. MicroRNA-183-96-182 Cluster Regulates Bovine Granulosa Cell Proliferation and Cell Cycle Transition by Coordinately Targeting FOXO1. Biol Reprod. 2016;94(6):127. 